\title{
HYPERCYCLICITY OF WEIGHTED TRANSLATIONS ON ORLICZ SPACES
}

\author{
M. R. AZIMI AND I. AKBARBAGLU
}

Abstract. In this paper, we study the hypercyclicity of the weighted translation $C_{u, g}$ defined on Orlicz space $L^{\Phi}(G)$ where $G$ is a locally compact group, $g \in G$ and $u$ is a weight function on $G$. It is shown that when $g \in G$ is a torsion element, then $C_{u, g}$ cannot be hypercyclic. However, for an aperiodic element $g \in G$, necessary and sufficient conditions for $C_{u, g}$ and its adjoint are given to be hypercyclic.

Mathematics subject classification (2010): 47A16, 46E30.

Keywords and phrases: Hypercyclic, weighted translation, locally compact group, Orlicz space.

\section{REFERENCES}

[1] S. I. ANSARI, Hypercyclic and cyclic vectors, J. Funct. Anal. 128 (1995), no. 2, 374-383.

[2] F. Bayart, É. Matheron, Dynamics of linear operators, Cambridge Tracts in Mathematics, Cambridge University Press, Cambridge, 2009.

[3] C. Chen, C.-H. Chu, Hypercyclic weighted translations on groups, Proc. Amer. Math. Soc. 139 (2011), no. 8, 2839-2846.

[4] C. Chen, C.-H. ChU, Hypercyclicity of weighted convolution operators on homogeneous spaces, Proc. Amer. Math. Soc. 137 (2009), no. 8, 2709-2718.

[5] K.-G. Grosse-Erdmann, A. P. Manguillot, Linear chaos, Universitext, Springer, London, 2011.

[6] C. KitAI, Invariant closed sets for linear opeartors, Thesis (Ph. D.), University of Toronto (Canada), 1982.

[7] M. M. RaO, Z. D. Ren, Theory of Orlicz spaces, Monographs and Textbooks in Pure and Applied Mathematics, 146, Marcel Dekker, Inc., New York, 1991.

[8] H. N. SAlas, Hypercyclic weighted shifts, Trans. Amer. Math. Soc., 347 (3) (1995) 993-1004. 